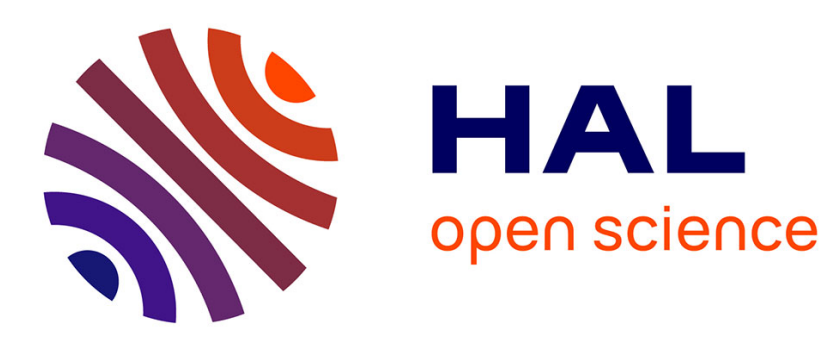

\title{
Le prix de la qualité. L'Etat et le cinéma français (1960-1965)
}

Frédéric Gimello-Mesplomb

\section{To cite this version:}

Frédéric Gimello-Mesplomb. Le prix de la qualité. L'Etat et le cinéma français (1960-1965). Politix, 2003, Politiques du cinéma, 16 (61), pp.95-122. 10.3406/polix.2003.1258 . halshs-01002341

\section{HAL Id: halshs-01002341 https://shs.hal.science/halshs-01002341}

Submitted on 10 Aug 2018

HAL is a multi-disciplinary open access archive for the deposit and dissemination of scientific research documents, whether they are published or not. The documents may come from teaching and research institutions in France or abroad, or from public or private research centers.
L'archive ouverte pluridisciplinaire HAL, est destinée au dépôt et à la diffusion de documents scientifiques de niveau recherche, publiés ou non, émanant des établissements d'enseignement et de recherche français ou étrangers, des laboratoires publics ou privés. 


\section{Le prix de la qualité. L'Etat et le cinéma français (1960-1965)}

Frédéric Gimello-Mesplomb

\section{Citer ce document / Cite this document :}

Gimello-Mesplomb Frédéric. Le prix de la qualité. L'Etat et le cinéma français (1960-1965). In: Politix, vol. 16, n61, Premier trimestre 2003. Politiques du cinéma. pp. 95-122;

doi : https://doi.org/10.3406/polix.2003.1258

https://www.persee.fr/doc/polix_0295-2319_2003_num_16_61_1258

Fichier pdf généré le 19/07/2018 


\title{
Résumé
}

Le prix de la qualité. L'Etat et le cinéma français : 1960-1965

Frédéric Gimello-Mesplomb

Le 28 mai 1946 à Washington, les accords Blum-Byrnes limitent à quatre par trimestre les semaines d'exploitations des films français de première exclusivité, une mesure qui mobilise l'ensemble de la profession et qui contribuera au développement d'un profond sentiment antiaméricain. Durant les deux années qui vont suivre, grèves et lobbying professionnel poussent l'Etat à intervenir dans le débat. Une loi est votée en 1948 instaurant un « fonds de soutien » à l'industrie cinématographique. Le fonds est alimenté par le prélèvement d'une fraction de 10,9\% du prix du billet, dite TSA (Taxe supplémentaire additionnelle), et sa principale mission est la redistribution de l'argent au secteur de la création. Ce système, toujours en vigueur, connaîtra des fortunes diverses sans que son principe soit remis en cause. Avec la mise en place de l'Avance sur recettes en 1959, le point d'achoppement des critères de sélection se noue désormais autour de la notion de qualité qu'entend encourager l'aide. Les années 1960 voient apparaître sur les fonds publics une esthétique officielle avantageant la qualité « standard » des cinéastes aguerris, offrant ainsi une vitrine à des films sensés jouer un rôle sur la scène de la diplomatie culturelle francophone. Un véritable réseau symbolique se créera au fil des années autour de l'obtention d'une subvention publique, cette dernière servant de gage aux films portant les valeurs culturelles partagées par les milieux socioprofessionnels les mieux représentés au sein des commissions décisionnelles : ceux du cinéma et des lettres.

\begin{abstract}
The Price of the Quality. The State and the French Cinéma: 1960-1965

Frédéric Gimello Mesplomb

May 28,1946 in Washington, the Blum-Byrnes agreements limit to 4 per quarter the weeks of exploitations of French films, a measure dissatisfied by the profession contributing to the development of a deep feeling anti-American. During the following two years, strikes and professional lobbying push the State to arbitrate the debate. A law is voted in 1948 founding "special funds of support" for cinema industry. The funds is fed by a tax on a fraction of $10,9 \%$ of the cinema ticket amount, and its principal mission is the redistribution of the money to the sector of cinema creation. This system is always in use. With the installation of the "Advance on receipts" (Avance sur recettes) in 1959, the point of obstacle into the criteria of selection is the definition of the concept of quality. Thanks to the public funds, the 60 's see appearing a official esthetics favouring a "standard" quality into films, offering a window to films prepared to play a role on the scene of French-speaking cultural diplomacy. A symbolic network will be created to obtain a public subsidy. The state financial support is a pledge of quality for films carrying the cultural values shared by French socio-professional decision-makers of cinema and letters.
\end{abstract}




\title{
Le prix de la qualité
}

\section{L'Etat et le cinéma français (1960-1965)}

Frédéric GIMELLO-MESPLOMB

\begin{abstract}
"Le cinéma français qui compte, c'est-à-dire un très petit nombre de films, qui sont presque toujours des œuvres de jeunes $[\ldots]$, une sorte de cinéma d'amateur d'une certaine génération à laquelle nous devons absolument donner tous les moyens. "
\end{abstract}

André Malraux, Discussion sur le budget de la Culture, Assemblée nationale, 14 octobre 1965

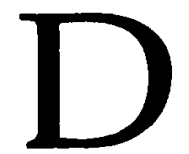

epuis la fin de la seconde guerre mondiale, l'intervention de l'Etat dans le soutien à la création cinématographique a pris la forme de subventions ou d'incitations financières directes destinées à encourager le talent et à soutenir les efforts pour le faire connaître. Dans les deux cas, c'est la notion de qualité qui est mobilisée afin d'effectuer le choix des œuvres de cinéma devant ou non être soutenues par l'Etat ou ses filiales. La détermination de cette qualité passe par un processus de sélection prévu par le code de la cinématographie et résulte de l'action de commissions d'experts, composées de "simples » spectateurs, de représentants de la profession et de l'administration, de représentants syndicaux. Chaque année apporte son lot de réalisateurs « recalés » à l'avance sur recettes, mais aussi sa quarantaine de films soutenus par l'argent de l'Etat et par les structuresrelais mises en place par le Centre national de la cinématographie (CNC) au 
profit des films bénéficiaires (Bureau d'accompagnement au premier film, aides à la distribution, festivals, implication de diffuseurs hertziens ou de chaînes thématiques).

Rendre compte de la politique publique de soutien au cinéma français implique donc d'analyser les mécanismes qui ont contribué, au fil du temps, à fixer les canons d'une culture cinématographique $d^{\prime}$ Etat et à modeler l'image de la production cinématographique nationale en distinguant des œuvres dignes d'être subventionnées par les pouvoirs publics de celles qui ne nécessitent pas son soutien ${ }^{1}$. C'est ainsi que nous avons essayé de dégager les critères de la qualité cinématographique promus par l'Etat à travers les orientations prises par les politiques de soutien à la création et plus précisément par l'avance sur recettes, modèle par excellence illustrant la politique de soutien mise en place par la France au service du cinéma. Après un rappel des principales mesures qui ont forgé cette politique de soutien au cinéma français, nous présenterons quelques résultats illustrant le " goût " de l'Etat en matière cinématographique, plus particulièrement à la lumière des cinq premières années de fonctionnement du système de l'avance sur recettes (1960-1965).

\section{Aux origines de la qualité nationale : valeur culturelle (française) contre-succès commercial (américain)}

\section{Les accords Blum-Byrnes et le développement de l'« anti-américanisme corporatif "}

En 1944, le cinéma français est contrôlé par le COIC (Comité d'organisation de l'industrie cinématographique), organisme régi par des professionnels répartis en différentes commissions spécifiques. Rapidement rebaptisé à la Libération OPC (Organisme des professionnels du cinéma), il prendra finalement le nom de CNC par ordonnance du 25 octobre 1946, en gardant un mode de fonctionnement très voisin des précédentes institutions. Cet héritage explique la subsistance de principes de fonctionnement marqués par la tendance corporatiste d'origine ${ }^{2}$. L'ordonnance de 1946 confère d'autre part au CNC une compétence accrue, l'organisme devenant

1. C'est en se penchant plus particulièrement sur les causes des sentiments pro-américains et anti-américains au sein des groupes professionnels du cinéma français, ainsi que sur la relation que ces sentiments entretiennent avec la conscience identitaire nationale, et notamment avec la notion d'œuvre "d'inspiration française ", que l'on saisit également l'intérêt offert par l'étude des politiques de soutien mises en place au cours des cinquante dernières années.

2. Certaines décisions du COIC furent conservées à des fins civiles, par exemple le décret relatif aux conditions d'attribution des cartes professionnelles, d'origine antisémite, régissant depuis 1940 les conditions d'entrée des techniciens dans la profession. 
administrativement et financièrement autonome, quoique encore rattaché statutairement à la tutelle d'un ministère ${ }^{3}$.

Le renouveau humaniste issu de la Résistance, l'exigence collective $d$ 'une plus grande justice sociale et la crainte $d^{\prime}$ une nouvelle manipulation des masses au détriment de la démocratie expliquent l'attention portée par les autorités au mouvement d'Education populaire. L'idée d'«éduquer le peuple » apparaît comme une nécessité incontournable au centre de laquelle on trouve une attention particulière portée au cinéma, art « populaire » par excellence. Les premières organisations de masse du spectateur "éclairé » (les ciné-clubs) apparaissent parallèlement au souci de l'Etat de développer la démocratisation culturelle et de mettre progressivement en œuvre une politique de soutien à la création cinématographique. Le milieu des professionnels du cinéma adhère aux revendications. Un premier projet de loi d'aide voit le jour ; mais il est retiré en mars 1946 sous la pression des exploitants, moins sensibles aux revendications sociales des créateurs et surtout attachés à conserver le contrôle des recettes. Le 10 avril 1945, en effet, Philippe Acoulon, dernier directeur du COIC, avait tenté d'instaurer un prélèvement de $7 \%$ sur le prix des billets dans le but d'alimenter un fonds de soutien, dont les usages ne sont alors pas encore clairement définis.

Malgré son échec, la tentative emporte l'adhésion d'un certain nombre de professionnels et de parlementaires. Les accords Blum-Byrnes, qui surviennent moins de trois mois après l'annulation du décret, ne manquent pas de rappeler à tous la nécessité d'un soutien financier de l'Etat. Signé le 28 mai 1946 à Washington par James Byrnes et Léon Blum, alors ambassadeur extraordinaire du Gouvernement provisoire, cet accord comporte un volet relatif à l'importation des films américains en France. Au soir du 29 mai, la presse française est unanime pour souligner le succès de la démarche. Les Etats-Unis annulent par cet accord commercial la totalité des dettes de guerre françaises et offrent à la France un crédit de 1370 millions de dollars. Pourtant, lors de l'annonce de l'annexe de deux pages intitulée « Arrangement entre le Gouvernement provisoire de la République française et le gouvernement des Etats-Unis d'Amérique au sujet de la projection des films américains en France " et portant sur la création, en France, d'un quota de quatre semaines par trimestre réservé au cinéma français, les avis divergent jusqu'à devenir rapidement hostiles au traité. Carné, Blanchard, Becker ou Laroche sont parmi les premiers artistes et critiques de cinéma à se mobiliser en dénonçant le «bradage du cinéma français ». Un sentiment

3. Les aléas de ce rattachement en disent d'ailleurs long sur la vision du cinéma qu'a le pouvoir politique avant 1959: ministère de l'Information en 1946 (du fait des actualités filmées, contrôlées par la censure), puis ministère du Commerce à partir de 1947, sans compter les différents services du cinéma existant alors au sein des ministères ou secrétariats d'Etat à l'Intérieur, aux Beaux-Arts, jusqu'à celui, célèbre et considérablement agencé, du ministère de l'Agriculture. 
d'abandon se répand au sein de la profession, sans que le phénomène puisse toutefois provoquer l'union des tendances syndicales. Dès le 5 juin, $L^{\prime} E c r a n$ français titre: "Une nouvelle période s'ouvre pour le cinéma français : celle de la lutte contre l'anémie menaçante et l'étouffement progressif ${ }^{4}$. " Les prémices d'un mouvement apparaissent autour du Syndicat français des producteurs de films (animé par Borderie, Hunebelle et Richebé) et de la Fédération nationale du spectacle CGT. Cette dernière a saisi les enjeux en présence et entend bien jouer la carte du social. A la mi-juin, une conférence de presse organisée par la Fédération à l'Institut des hautes études cinématographiques (IDHEC) dénonce les conséquences néfastes de l'accord en termes de chômage (que l'Humanité du 10 juin 1946 évalue à un ordre de quarante à quatre-vingt mille emplois de techniciens). Président d'une autre organisation syndicale (l'Association des auteurs de films), Raymond Bernard donne le ton d'un discours qui sera maintes fois repris dans les décennies suivantes: "Depuis la découverte de l'imprimerie, les hommes n'ont jamais disposé d'un véhicule de pensée plus puissant. C'est pourquoi le visage de la France ne doit pas être effacé. Il faut que notre cinéma subsiste ${ }^{5}$.

En revanche, si la profession se trouve momentanément soucieuse de bénéficier du soutien de l'Etat, elle ne souhaite pas un interventionnisme trop rigide. Le clivage se situe au niveau du maintien d'une souplesse de trésorerie, point revendiqué par les producteurs, les distributeurs et les exploitants de salles. En 1995, Pierre Billard note à ce sujet que :

"Les ultras du libéralisme souhaitent que l'intervention de l'Etat se manifeste, au pire, par le biais d'une "direction ministérielle" qui assurerait la coordination avec la Confédération générale du cinéma, elle-même chargée de contrôler la profession. [...] Car lorsque Roger Weil-Lorac, qui fut le délégué général très consciencieux de la Confédération générale du cinéma, évoque dans ses souvenirs (Cinquante ans de cinéma actif) les débats de cet organisme, il apparaît que la principale revendication des exploitants est d'exercer euxmêmes le contrôle sur les recettes, tandis que celle des producteurs est d'échapper au contrôle du financement des films ${ }^{6}$. »

Autre point de désunion: les distributeurs et les exploitants envisagent plutôt favorablement l'arrivée de films américains (réalisant davantage d'entrées que les films français), tandis que les producteurs partent en ordre dispersé, faisant appel à l'aide de l'Etat pour faire face à l'ouverture, même partielle, de la concurrence. Si bien que les accords Blum-Byrnes sur le

4. L'écran français, 49, 1946, p. 1.

5. Hubert-Lacombe (P.), Le cinéma français dans la guerre froide (1946-1956), Paris, L'Harmattan, 1996, p. 27.

6. Billard (P.), L'âge classique du cinéma français, Paris, Flammarion, 1995, p. 504. 
cinéma ne suscitent pas l'émotion que leur a associée a posteriori leur « légende noire ${ }^{7}$ ".

Après une année 1947 morose, passée dans l'attentisme, ce sont les premières conséquences sociales des accords, largement commentées par Georges Sadoul dans L'Humanité, puis par Pierre Kast dans Action et Henri Magnan dans Le Monde, qui vont finalement aider les professionnels à prendre conscience de leur réelle portée. Cette prise de conscience, bien que tardive, trouve un puissant relais dans l'action du Parti communiste qui, après la fin du tripartisme, mobilise ses réseaux dans les milieux culturels et se trouve à l'initiative de la création du Comité de défense du cinéma français $(C D C F)$, réunissant autour des réalisateurs Marcel L'Herbier et Claude Autant-Lara, des syndicalistes et des producteurs. Les débuts de l'année 1948 voient le CDCF mener une campagne tous azimuts tandis que l'on assiste à une radicalisation des propos, à l'image de la motion votée par André Berthomieu et le Syndicat des techniciens (Fédération nationale des artisans du film) : "Fort du constat que le gouvernement n'a pas encore pris en considération la situation désespérée, et que "rien ne change", le Syndicat $s^{\prime}$ engage à défendre jusqu'au bout l'art cinématographique français ${ }^{8}$. " A ces exhortations qui s'apparentent davantage à des mots d'ordre sans espoir, la Fédération du spectacle CGT, présidée par Claude Autant-Lara, appelle quant à elle à descendre dans la rue :

"La seule voie possible, c'est celle de la manifestation du 4 janvier sur les grands boulevards. C'est la création dans toute la France de comités de défense du cinéma français. Il faut imposer aux responsables de ce pays l'élaboration rationnelle d'une véritable politique française du cinéma. Qu'il reprenne sa place et qu'il conserve son indépendance dans le monde9. "

C'est donc finalement plus d'un an et demi après la signature des accords Blum-Byrnes que leur remise en cause par la profession se généralise. La popularité de la campagne atteint son apogée lors de l'organisation par le CDCF de cette manifestation sur les grands boulevards, le 4 janvier 1948. Le cortège rassemblera plusieurs dizaines de milliers de participants, dont la mémoire collective retiendra les visages des vedettes du moment: Jean Marais, Simone Signoret, Jacques Becker, Jean Grémillon, Louis Daquin, etc. Attaqué pour avoir bradé le cinéma français à l'Amérique de Truman, le gouvernement fait machine arrière. L'ouverture $d^{\prime} u n$ nouveau round de négociations aboutit, le 16 juillet 1948, à la signature de nouveaux accords. D'une durée de quatre ans, ces accords reviennent sur l'épineux problème

7. Portes (J.), « Les origines de la légende noire des accords Blum-Byrnes sur le cinéma », Revue d'histoire moderne et contemporaine, 2, 1986 et Jeancolas (J.-P.), "L'arrangement Blum-Byrnes à l'épreuve des faits: les relations (cinématographiques) franco-américaines de 1944 à 1948 ", Revue de l'association française de recherche sur l'histoire du cinéma (AFRHC), décembre 1993.

8. Le spectacle, 10, 1948.

9. Ibid. 
des quotas. Les films français obtiennent désormais une période d'exploitation garantie de vingt semaines par an au lieu de seize, tandis qu'est institué un contingentement de distribution, fixant à un maximum de 121 le nombre annuel de films américains doublés autorisés à être distribués sur le territoire français.

Mais surtout l'Etat ressort le projet d'une aide automatique. Votée à l'initiative du député SFIO Géraud-Jouve en septembre 1948, la « loi d'aide temporaire à l'industrie cinématographique " va poser les bases de ce qui est aujourd'hui considéré comme l'un des piliers de l'exception culturelle française. Le système énoncé est celui d'un prélèvement général sur tous les billets de cinéma vendus en France (y compris sur ceux des films étrangers distribués sur le territoire hexagonal et dans les colonies) d'une fraction de $10,9 \%$ du prix du billet, dite TSA (Taxe supplémentaire additionnelle). Cette taxe alimente un fonds spécial, le Fonds d'aide à l'industrie cinématographique (ou Fonds de soutien) dont la principale mission est la redistribution de l'argent au secteur de la création, selon des grilles mathématiques (au prorata des recettes engendrées par les films précédemment réalisés). L'aide est ainsi dite " automatique ». Le Fonds de soutien a depuis connu des fortunes très diverses. En 1948 il n'est alors alimenté que par la TSA et une imposition financière sur le métrage des films (français et étrangers) à leur sortie dans les salles. $\mathrm{Si}$, aujourd'hui, son financement comporte toujours une part non négligeable de TSA, sont venues depuis $s^{\prime} y$ ajouter des taxes sur les sociétés de diffusion de programmes audiovisuels (chaînes de télévision), des taxes sur les chiffres d'affaire de publicité de ces mêmes sociétés, une taxe sur le produit des abonnements (chaînes thématiques), auxquelles s'ajoutent des dotations directes du ministère de la Culture.

\section{Du Fonds de développement à l'avance sur recettes : la définition de la qualité}

Pour sa première année de fonctionnement, la loi temporaire de 1948 permet au CNC de recueillir quelque 1952 millions d'anciens francs, dont 1671 proviennent de la TSA. La première répartition des recettes affirme un net penchant pour le secteur de la production (800 millions), devant le secteur de l'exploitation ( 250 millions) et les actualités filmées ( 80 millions). Mais les premiers désagréments du système ne tardent pas à se faire sentir. Le mécanisme de redistribution des recettes à la production encourage une sorte de circuit fermé au sein duquel les projets de films visant les salles commerciales s'assurent avant tournage un confortable autofinancement, légitimant ainsi les recettes esthétiques et les ressorts dramatiques du fameux "cinéma de papa ». Autrement dit, comme le note André Bazin, l'institution d'un fonds de soutien provoque l'effet inverse de celui qui était initialement recherché, l'argent attirant l'argent : 
"Dans l'état actuel des choses, un producteur qui ne prend pas de risque et engage la dentition de Fernandel pour un nouveau Barnabé est sûr de gagner deux fois et de voir l'argent du Fonds d'aide s'ajouter à celui des spectateurs. Le producteur qui cherche à "faire de la qualité" court a priori un double risque virtuel : d'abord celui inhérent à la qualité (laquelle coûte souvent cher), ensuite celui de voir se dérober le pactole supplémentaire espéré. Concluons : sous couleur d'une justice approximative mais pratique, l'actuelle application de la Loi d'aide constitue automatiquement une prime au moindre risque, c'est-à-dire à la médiocrité10. »

Le législateur intervient à nouveau dans le débat. Le 6 avril 1953, à la suite de critiques répétées émanant de cercles de cinéphiles, le CNC adjoint à titre expérimental aux critères automatiques en vigueur celui de la "qualité " pour le court métrage, lui réservant $10 \%$ du Fonds de soutien ${ }^{11}$. Le film n'est dès lors plus un produit uniquement commercial, on lui confère évolution de taille - une valeur esthétique intrinsèque. En 1953, la France produit environ quatre cents courts métrages par an ${ }^{12}$. Cette décision aura une influence considérable sur la production française puisque la mesure permet à de nombreux jeunes gens, fraîchement émoulus de l'IDHEC (Resnais), issus de la photo (Varda, Demy), anciens assistants-réalisateurs (Ménegoz, Camus, Baratier) ou réalisateurs de films industriels (Fabiani, Molinaro) d'accéder enfin à l'univers du court métrage «artistique », tel qu'on l'appelait alors. Le «Groupe des trente » (formé de trente nouveaux cinéastes ayant tourné leur premier film en un laps de temps assez réduit) trouve dans cette prime à la qualité un marchepied artistique ainsi que la légitimation d'un statut d'auteur qui sera officiellement reconnu par les articles relatifs aux œuvres audiovisuelles de la loi du 11 mars 1957.

La mise en place de la prime à la qualité provoque l'arrivée des commissions d'experts chargées de définir la qualité des films. A l'initiative d'Ambroise Roux, directeur de cabinet de Jean-Marie Louvel, ministre de tutelle du cinéma (Industrie et Commerce), est créé conjointement à la réforme de 1953 un Conseil supérieur du cinéma qui fonctionnera jusqu'en 1960. Présidé par Guy Desson, ce conseil est composé de cinq collèges dont les deux premiers sont constitués de représentants des pouvoirs publics ${ }^{13}$ et les trois autres de représentants de professionnels ${ }^{14}$. Il revient à ce conseil de choisir les

10. Déclaration d'A. Bazin dans Radio-Télévision-Cinéma, 64, 1951, p. 6.

11. Rebaptisé pour la circonstance Fonds de développement.

12. Auparavant, seuls 150 courts métrages associés à la distribution d'un long métrage se voyaient rétribués financièrement, au prorata des recettes engendrées par le long métrage.

13. Deux représentants du Parlement, un représentant du ministère de l'Industrie et du Commerce, un représentant du secrétariat d'Etat à l'Information.

14. Employeurs : $\Lambda$ dolphe Trichet, Jean-Pierre Frogerais et Roger Sallard (suppléant). Salariés : Léon Carré, Raymond Le Bourre et Robert Florat (suppléant). Personnalités choisies pour leur compétence artistique ou financière en matière de cinématographie : Maurice Bessy (critique), Gabriel David (président du Crédit national) et Roger Weil-Lorac (suppléant), délégué de la Confédération nationale du cinéma français. 
premiers courts métrages bénéficiaires de la prime à la qualité. Ainsi, quatrevingts primes annuelles sont attribuées pour cent vingt candidats. La mise en place de ce dispositif ne se fait pas sans heurts ni sans débats. Le principal blocage est naturellement celui de la définition de la notion de qualité. En avril 1951, la Fédération du spectacle CGT, l'Association des auteurs de films et l'Association française de la critique de cinéma s'étaient déjà longuement penchées sur le sujet, afin de fournir au CNC les moyens de délibérer équitablement pour la délivrance des visas de tournage :

"Faut-il définir la qualité ? Si difficile, si délicat, aussi, que cela soit, il se pourrait pourtant qu'une seule définition, une fois juridiquement articulée, soit le seul moyen de conférer à la commission, par référence à un texte accepté, l'indispensable autorité sur laquelle elle devra se fonder. Il existe le danger contraire : celui qu'elle en soit la prisonnière. L'important est donc en définitive que toute liberté d'appréciation soit en fait laissée à la commission, et qu'elle soit compétente ${ }^{15}$. "

Une conclusion alambiquée qui ne satisfait guère les parties. Pour les critiques et les cinéphiles qui s'expriment dans les revues à destination du public cultivé, comme Les cahiers du cinéma et Positif, le critère de "l'auteur » apparaît le plus apte à mesurer et à anticiper la qualité du film.

Quelques mois après les décrets des 30 avril et 20 mai 1955 qui étendent le principe de « la prime à la qualité » des courts métrages aux longs métrages, la rédaction de Positif rappelle que "le critère de la qualité " demeure "le cœur du problème ». Dépendant de « la composition du jury » qui l'attribue, il sera suspendu aux jugements de ce dernier :

"Nous aurons la qualité de ce jury", note Positif, qui espère que ce dernier " ne comprendra pas trop de figurants décoratifs, d'amateurs de belles photos et interprétations sobres sur fond de scénarios bien construits, ni trop de représentants de grandes familles spirituelles et de courants d'opinions pour redressement moral ou politique. Nous osons espérer que la qualité primée ne sera pas "la qualité française" de M. Delannoy. Car il est inutile de persuader des producteurs qui réalisent une affaire sûre, qu'ils sont en même temps des mécènes ${ }^{16}$. "

Tout en réaffirmant ses positions politiques « marxisantes " et anticléricales qui la distinguent de sa rivale parisienne (Les cahiers du cinéma), la revue n'en reprend pas moins la thèse dénonciatrice de « la qualité française " défendue un an plus tôt par François Truffaut et les jeunes turcs des Cahiers du cinéma - thèse dont on sait qu'elle préfigure la proposition positive d'une "politique des auteurs » et la justification théorique de la Nouvelle Vague ${ }^{17}$.

15. Cité dans Radio-Télévision-Cinéma, 64, 1951, p. 6.

16. Positif, 14-15, 1955, p. 162-163.

17. Le " cinéma de la qualité française » est une expression ironique utilisée par $F$. Truffaut à des fins polémiques pour dénoncer un cinéma à la fois académique et coûteux, marqué par 
L'argument traditionnel qui oppose le producteur soucieux de rentabilité financière au mécène désintéressé traduit le scepticisme de la revue quant à la nouveauté d'une mesure qui rompt difficilement avec le mécanisme déjà en place d'une " prime au succès " commercial. D'où la proposition concrète de Positif :

"La part d'aide obtenue par un film pourrait être attribuée non seulement à son producteur, mais à ses auteurs (réalisateurs et scénaristes), pour financer leurs œuvres suivantes. On voit immédiatement les possibilités d'un tel système : les auteurs deviennent coproducteurs. Quelques millions peuvent leur permettre d'imposer un scénario intelligent, un acteur inconnu, etc. Voilà de quoi non seulement soutenir les cinéastes consacrés, mais aussi de réveiller le courage de certains autres, résignés au commerce ${ }^{18}$."

André Bazin, fondateur et rédacteur en chef des Cahiers du cinéma, défend une position équivalente avec des arguments identiques :

"La principale raison invoquée par la Confédération générale du cinéma français [...], c'est l'impossibilité juridique de définir et de détecter la qualité. Dans ces conditions, la recette d'un film serait le critère le moins arbitraire, puisqu'il témoigne au moins de la satisfaction du public. Le caractère pragmatique de cet argument en fait sa force. Il est pourtant loin d'être irréfutable. [...] Il est vrai que de bons films comme La symphonie pastorale ou Justice est faite peuvent réussir financièrement tout comme Le tampon du capiston, L'épave, ou La maison du printemps. Mais le palmarès des chefsd'œuvre tombés au champ d'honneur de l'exploitation cinématographique est là pour témoigner des risques de la qualité. Ni L'Atalante, ni Le crime de Monsieur Lange, ni La règle du jeu, ni Le ciel est à vous, ni Les dames du Bois de Boulogne, pour ne citer que ceux-là, n'ont été des succès financiers, quand ils n'ont pas même constitué pour le producteur une catastrophe financière. [...] Nous souhaitons tous le succès du Journal d'un curé de campagne, mais il se pourrait qu'il connût le sort des Dames du Bois de Boulogne. Ce sont des déboires auxquels ne s'expose pas M. Cozinet en tournant Le don d'Adèle. Si l'attribution d'une aide a un sens, même comme une surprime au succès, on voit donc qu'elle ne devrait pas, en bonne équité, aller indifféremment à Justice est faite et à Caroline chérie19. "

Contournant le point de vue pragmatique qui reconnaît l'imprévisibilité du chef-d'œuvre et l'impossibilité d'identifier a priori la qualité future du film,

\footnotetext{
l'adaptation d'œuvres littéraires prestigieuses. Elle visait notamment les réalisateurs suivants : Delannoy, Autant-Lara, Christian-Jaque ainsi que les scénaristes Bost et Aurenche qualifiés de "Viollet-Leduc de l'adaptation " (Truffaut (F.), "Une certaine tendance du cinéma français", Les cahiers du cinéma, 31, 1954). Sur l'histoire de l'expression " qualité française » et de l'article qui la vulgarise ainsi que sur "la politique des auteurs", cf. De Baecque (A.), Les cahiers du cinéma. Histoire d'une reoue, tome I, Paris, Editions Cahiers du cinéma, 1991, p. 99-104 et Marie (M.), La Nouvelle Vague, une école artistique, Paris, Nathan, 1997, p. 31-43.

18. Positif, 14-15, 1955, p. 164.

19. Déclaration d'A. Bazin dans Radio-Télévision-Cinéma, 64, 1951, p. 6.
} 
André Bazin propose donc, au nom de l'équité, de réserver les fonds publics aux films dont la rentabilité est faible compte tenu de leur sujet, et de donner à l'Etat un rôle de moralisation de la production cinématographique en soutenant publiquement les films ambitieux culturellement. Le décret d'application de la nouvelle prime se fait l'écho de cette préoccupation en proposant une formule de compromis entre le critère de l'identité nationale, des critères de créativité et des critères éducatifs. Il prévoit en effet d'aider les films « de nature à servir la cause du cinéma français, ou à ouvrir des perspectives nouvelles à l'Art cinématographique, ou à faire connaître les grands thèmes et problèmes de l'Union française " (art. $28 \mathrm{du}$ code de l'industrie cinématographique). Cette définition va dicter les délibérations du Conseil supérieur du cinéma durant près de six ans.

\section{Experts et expertise du film de qualité}

\section{La création de l'avance sur recettes}

La prime à la qualité est donc élargie aux longs métrages en mai 1955. Quelques cinéastes des années précédant la Nouvelle Vague en bénéficient : Alexandre Astruc (Le rideau cramoisi), Jacques Baratier (Goha le simple), Marcel Camus (Mort en fraude), Louis Malle (Ascenseur pour l'échafaud), Pierre Kast (L'amour de poche) ou Claude Chabrol (Le beau Serge et Les cousins). En mars 1959, tandis que le CNC passe sous la houlette du nouveau ministère des Affaires culturelles, il est question de moderniser l'attirail législatif en place autour de l'aide au cinéma. Ce sera le travail d'un homme, Pierre Moinot, un conseiller à la Cour des comptes, ancien de l'administration d'outre-mer, ayant rejoint quelques semaines plus tôt le cabinet Malraux au poste de conseiller technique pour le cinéma. Pierre Moinot consulte les représentants des organisations professionnelles et, aidé du directeur-adjoint du CNC, Jean Gründler, rédige en quelques semaines une nouvelle réglementation qui paraît dans le décret modifié du 16 juin. Le poids de la toute jeune Communauté économique européenne a plané sur la rédaction des articles : tout l'art des deux hommes a été de contourner les directives de Bruxelles (notamment l'interdiction de concurrence vis-à-vis d'autres cinématographies européennes) en insistant sur les motivations strictement « culturelles » de l'aide publique en faveur du cinéma.

Si les principes du Fonds de développement de 1953 sont conservés dans leurs grandes lignes éthiques et budgétaires, la grande nouveauté du décret modifié du 16 juin 1959 est la création de l'avance sur recettes, qui remplace la prime à la qualité tout en conservant un mode d'attribution identique (une commission, un vote, un film aidé). Restant encore flou quant aux critères de qualité souhaités par l'Etat, le décret souligne cependant que la mesure est décidée "pour faire face aux automatismes fondés sur le seul 
succès commercial ». Cette interprétation du succès commercial, entourée d'un certain nombre d'ambiguïtés, va creuser le premier écart tangible entre le cinéma marchand et le cinéma subventionné. Ce dernier va endosser l'image d'un cinéma voué à la recherche esthétique et au non-profit, alors que la loi de 1953 avait établi autour de la répartition des aides un aimable consensus entre producteurs et pouvoirs publics, la distinction entre le cinéma d'auteur et le cinéma commercial n'apparaissant pas du fait des critères d'attribution automatiques. En ce qui concerne les modalités de fonctionnement de l'avance sur recettes, le décret maintient le système de la commission d'experts :

« Des avances [...] sont accordées soit avant, soit après la réalisation du film, en fonction notamment de la nature du sujet, des caractéristiques et des qualités de l'œuvre cinématographique et des conditions de réalisation, après avis d'une commission réunissant des personnes compétentes sur le point de vue financier, technique et artistique ${ }^{20}$."

Un remboursement de cette avance sur recettes est initialement prévu. II devra s'effectuer en trois étapes: le «couloir», qui désigne le remboursement de l'avance avant amortissement total du film (le taux est de $5 \%$ des recettes nettes producteur); le remboursement " normal " de l'avance, une fois le film amorti, sur la recette nette producteur ${ }^{21}$ (le taux représente $50 \%$ ) ; l' « épargne forcée " (une fois l'avance remboursée, le CNC prélève $15 \%$ des recettes pour alimenter le compte de soutien automatique du producteur qui sera disponible pour le financement de son prochain film).

\section{Au cœur du système d'expertise}

En janvier 1960, lors de sa première réunion, la Commission de l'avance sur recettes, modestement dotée de 8,5 millions d'anciens francs, n'est qu'une émanation de la nouvelle Commission consultative du cinéma, qui remplace le Conseil supérieur du cinéma siégeant au CNC depuis 1953. Forte de ses 27 membres (fonctionnaires, professionnels et parlementaires), cette assemblée se réunit tantôt en sous-commission afin de délivrer les "visas préalables " (littéralement "sous-commission chargée d'émettre un avis sur les demandes d'agrément de films de long métrage"), tantôt en "souscommission d'attribution des avances ou garanties de recettes ». Dans ce cas,

20. Décret modifié du 16 juin 1959.

21. Pour le CNC, le film est considéré comme amorti lorsque la somme du total des recettes nettes et de l'aide automatique est égale à celle du montant du devis du film moins le montant de l'avance sur recettes. 
elle est assistée de membres nommés pour leur expertise artistique ${ }^{22}$. Ces experts sont choisis à la discrétion du ministre des Affaires culturelles parmi des personnalités proche du milieu littéraire ou parmi des " amis des arts ", alors que les membres représentant les traditionnels corps de métiers du cinéma sont proposés par les principaux représentants des branches syndicales au directeur du CNC. Comme les autres membres, les membres nommés détiennent un mandat d'une durée d'un an. En 1960, ils ont comme nom Jacques Audiberti, Jean Dutourd, Robert Kanters, Léon Mathot, Georges Magnane, Edgar Morin, Pierre Moinot (qui siège en qualité d'écrivain) et Henri Queffelec. Au cours des dix années qui suivent, d'autres viendront les rejoindre ou les remplacer: Julien Gracq, Marguerite Duras, Mathieu Galey, Jean d'Ormesson, René Billetdoux, Michel Mohrt, Milan Kundera, Yves Navarre ${ }^{23}$. Si bien que les premiers reproches faits à l'avance sur recettes ne concernent guère la façon de répartir les subsides de l'Etat, mais bien la qualité socioprofessionnelle des membres nommés, à qui l'on reproche tantôt le fait d'être "extérieurs à la profession" et "de rien comprendre aux problèmes de la corporation ", tantôt le fait d'appartenir au "Tout-Paris littéraire et politico-littéraire ${ }^{24}$ ».

Jusqu'en 1972, les premières sous-commissions de l'avance sur recettes seront présidées par des fonctionnaires de la Cour des comptes : PierreHenri Lichtemberger, puis Pierre Moinot (qui quitte en 1964 le collège des experts artistiques acquis en sa qualité d'écrivain pour présider l'avance sur recettes jusqu'en 1972). Le tableau ci-dessous est un instantané de l'état de la commission en janvier 1962. Il précise les noms des titulaires et des suppléants ainsi que le titre professionnel grâce auquel les membres peuvent siéger ${ }^{25}$ :

22. D'autres commissions du CNC vont s'élargir à des experts, comme celle chargée des festivals, qui fait intervenir plusieurs acteurs de la diplomatie culturelle ( $R$. Cravenne, secrétaire général d'Unifrance, par exemple).

23. Sans oublier Georges Kiejman, Michel Polac, Erick Orsena, et jusqu'à dernièrement l'éditeur Christian Bourgois, Philippe Djian, Bernard Henri-Lévy et Frédéric Mitterrand.

24. Tracts syndicaux (archives de l'auteur).

25. Cet élément a toute son importance. En dépouillant les listes publiées annuellement par le Journal officiel et en étudiant la composition des collèges professionnels, nous avons mis à jour un système de "chaises musicales " lié à la quête du maintien du pouvoir décisionnel par les producteurs : les syndicats de producteurs avaient pris l'habitude de "placer» des hommes cumulant plusieurs activités leur permettant de détenir une carte du CNC pour chacune d'entre elles et ainsi de siéger l'année suivante au titre d'un autre collège et/ou dans l'une ou l'autre des commissions existantes. Reviennent ainsi durant plus de dix ans les noms de F. Cosne, de G. Loureau ou de R. Ribadeau-Dumas (respectivement producteur, distributeur et représentant du Parlement). 


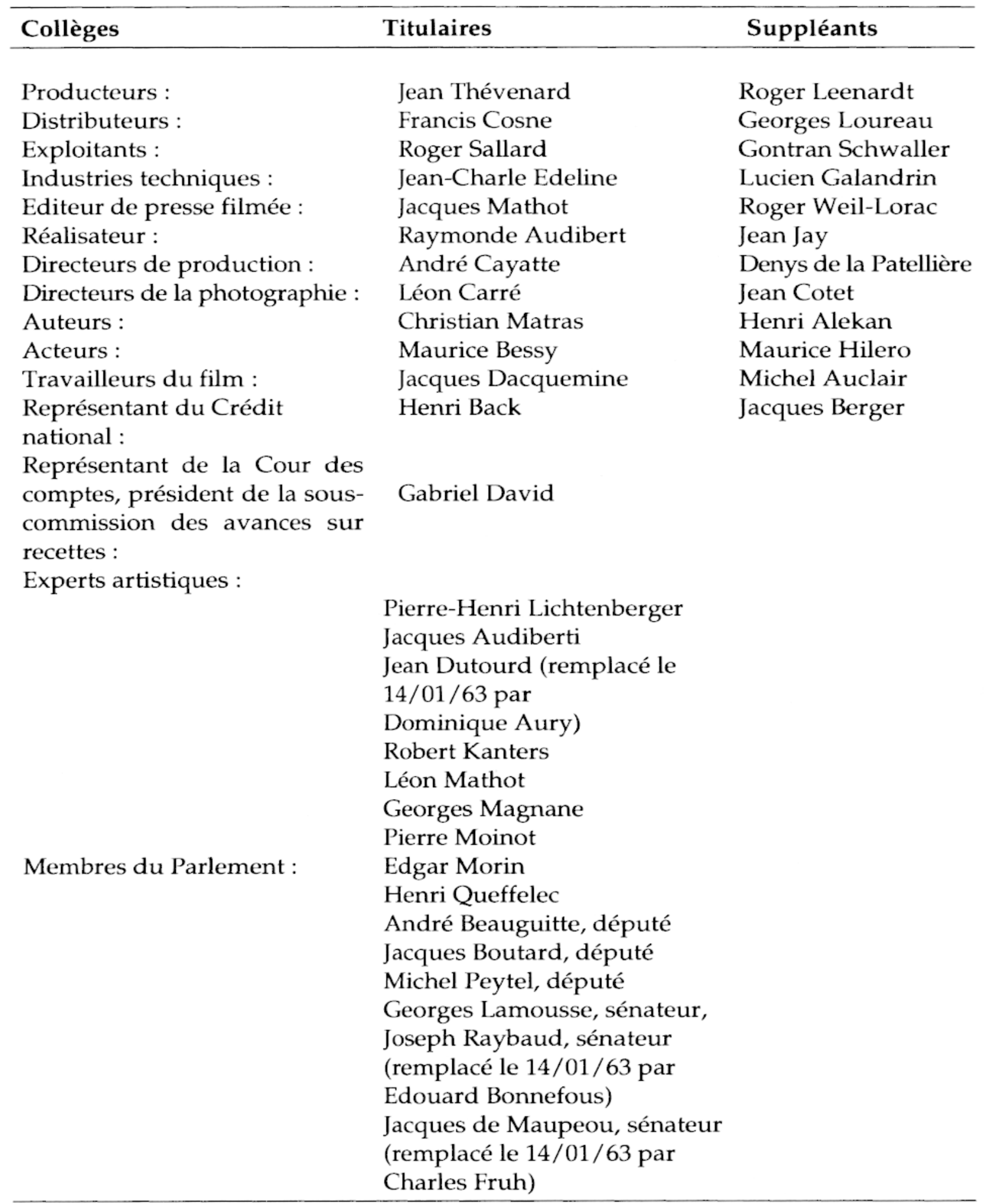

(Source : Journal officiel, 10 janvier 1962) 
Dès 1960, l'avance sur recettes adopte un principe singulier : pour postuler, le film doit généralement être terminé, le producteur devant déposer à l'enregistrement du secrétariat de la commission un dossier complet de demande d'avance, avec une copie du film ainsi que son numéro de visa de tournage. Les candidatures sont examinées dans l'ordre de dépôt, après visionnage des films par les membres. Les usages des commissions des années 1950, qui dispensaient les primes à la qualité après projection sont encore très présents. Il faudra attendre 1962 pour que l'avance sur recettes s'ouvre plus largement aux projets sur scénario, ce qui, sauf exceptions, constitue aujourd'hui son fonctionnement habituel. A partir de 1962, le nombre de projets sollicitant une avance sur scénario augmente donc et les procédures de demande de visa sont groupées avec celles de l'avance sur recettes. Dans les faits, la commission consultative peut délivrer un visa préalable mais refuser une avance, comme elle peut très bien accorder les deux. Le regroupement de ces deux fonctions entraîne naturellement les demandeurs à vouloir faire " coup double ", c'est-à-dire à décrocher avec le visa de tournage une avance substantielle afin de gagner du temps dans le montage financier de leur projet. Le président et les membres de la Commission de l'avance sur recettes ont toute latitude pour fixer leur choix sur tel ou tel type de film. Les décisions de soutien ou de rejet sont sans appel.

\section{La liste du CNC}

La liste annuelle des films bénéficiaires, longtemps tenue secrète, fut rendue pour la première fois publique en 1969 dans le bulletin annuel du CNC, sous la pression de plusieurs réalisateurs, avec une première liste de près de trois cents noms et de titres de films, sans chiffres. Dans les années qui suivront, le CNC publiera annuellement (au mois de mai) une très laconique liste de films, sans noms de réalisateurs et, jusqu'en 1973, sans indications concernant les montants alloués. Il faut attendre 1982 pour avoir communication d'une liste complète mentionnant les noms des réalisateurs bénéficiaires, les titres des films et le montant des avances. Au gré des alternances politiques des années 1980, la liste annuelle des bénéficiaires se fera à nouveau plus opaque; le principe de transparence ne se généralisera finalement qu'à partir de 1991. Ces hésitations conjoncturelles ne sont qu'un exemple illustrant le tabou qui pèse, depuis 1959, sur le cinéma français dès lors qu'il s'agit de définir quel est le choix qualitatif de l'Etat en matière d'aide au cinéma, mais plus encore quand il s'agit de le faire savoir.

Dans un travail récent ${ }^{26}$, nous avons tenté de cerner l'esthétique d'Etat à travers un corpus constitué des films français bénéficiaires d'une avance sur

26. Gimello-Mesplomb (F.), La politique des aides d'Etat entre 1960 et 1965 : de la Nouvelle Vague à l'entretien d'un cinéma d'évasion, DEA, Université Toulouse 2, 1997 et Gimello- 
recettes entre 1960 et 1965 . Cette démarche nous invitait à une nécessaire et indispensable «économie politique " du fait cinématographique, car cette période est celle qui voit éclore puis disparaître la Nouvelle Vague. Ce mouvement esthétique accouche $d^{\prime}$ un symbole fort: la notion d'indépendance artistique, dont le potentiel symbolique et l'idéologie qui la sous-tend prendront, dans les débats culturels des années 1980 et 1990, une importance considérable. Entre le $1^{\text {er }}$ janvier 1960 et le 31 décembre 1965, 192 films furent bénéficiaires d'une avance sur recettes, pour un montant moyen de 237042 anciens francs ${ }^{27}$, comme nous l'indique le tableau cidessous réalisé à partir des chiffres globaux :

$\begin{array}{lcccc}\text { Années } & \begin{array}{c}\text { Montants de la dotation } \\ \text { (anciens francs) }\end{array} & \begin{array}{c}\text { Nombre } \\ \text { de films } \\ \text { aidés }\end{array} & \begin{array}{c}\text { Total des } \\ \text { films produits } \\ \text { en France }\end{array} & \begin{array}{c}\text { Moyenne } \\ \text { par film } \\ \text { (anciens francs) }\end{array} \\ 1960 & 8500000(6360000 \text { utilisés) } & 22 & 158 & 289000 \\ 1961 & 9000000(9275000 \text { utilisés) } & 45 & 167 & 206111 \\ 1962 & 7500000(5185000 \text { utilisés) } & 27 & 125 & 192037 \\ 1963 & 9750000(5400000 \text { utilisés) } & 33 & 141 & 163636 \\ 1964 & 9750000(8270000 \text { utilisés) } & 30 & 148 & 275666 \\ 1965 & 8650000(10350000 \text { utilisés) } & 35 & 142 & 295714 \\ \text { Total } & 53150000 & 192 & 948 & 237042\end{array}$

Dans un premier temps, nous avons procédé à l'étude de ce corpus de 192 films sur un plan purement quantitatif. Nous avons procédé à des classements très simples par nombre de films aidés et par somme allouée selon divers critères (avant ou après passage devant la commission, etc.). Nous avons ainsi rapidement déterminé les cinéastes qui avaient eu le plus grand nombre de films soutenus durant cette période : quatre films (Jean Delannoy et René Clair) ; trois films (Etaix, Yves Robert, Granier-Deferre, Rossif, Franju, Resnais, Rouch, Enrico, Baratier, Mocky); deux films (Bresson, Melville, Gance, Cayatte, Lamorisse, Verneuil, Camus, Dreville, Alex Joffé, Ciampi, Hervé Bromberger, Lelouch, Vierne, Deville, Lautner, Drach, Agostini, Michel André, Varda, Colpi, Reichenbach, Truffaut, DoniolValcroze, De Broca, Molinaro).

Mesplomb (F.), Enjeux et stratégies de la politique de soutien au cinéma français, Thèse en études cinématographiques, Université Toulouse 2, 2000.

27. L'aide automatique, engagée sur un film au prorata des recettes précédentes, pèse en ces années-là entre $20 \%$ et $40 \%$ du budget moyen (en 1963, Truffaut réalise La peau douce, film estimé à 1370000 francs, avec un soutien automatique de 700000 francs) et l'avance sur recettes entre $20 \%$ et $35 \%$ (entre $3 \%$ et $5 \%$ en 2001 ). L'avance sur recettes représente à elle seule $48 \%$ des aides qualitatives distribuées par le CNC en 1960 et 1961 et $40 \%$ en 1963. 
Les autres classements quantitatifs ont par exemple permis de déterminer les sommes les plus importantes versées par la commission après projection du film :

$\begin{array}{llll}1 & \text { Gance } & \text { Austerlitz } & 600000 \\ 2 & \text { De Broca } & \text { Cartouche } & 350000 \\ 3 & \text { Delannoy } & \text { Les amitiés particulières } & 300000 \\ & \text { De la Patellière } & \text { Un taxi pour Tobrouk } & 300000 \\ & \text { Cayatte } & \text { Le passage du Rhin } & 300000 \\ 4 & \text { Carné } & \text { Terrain Vague } & 250000\end{array}$

De la liste globale des 192 films lauréats, et après l'examen d'une importante bibliographie sur les courants esthétiques du cinéma français, nous avons procédé dans un second temps à des classements qualitatifs en regroupant les principaux bénéficiaires en quatre grandes tendances esthétiques: le cinéma de la Nouvelle Vague stricto sensu (le groupe de cinéastes ayant mûri à l'ombre des Cahiers du cinéma, vaste entreprise de réflexion dont André Bazin fut le père spirituel et les locaux de la revue à couverture jaune le foyer animé); le renouveau éthique, esthétique et économique du cinéma français des années 1960 (Resnais, Varda, Hanoun, Rouch, Marker, Cavalier, etc.) appelé ici "nouveau cinéma " (autre dénomination de la tendance "rive gauche" en raison de l'appartenance politique de ses principaux auteurs) ; le cinéma de grande production commerciale et de prestige basé sur une image travaillée et un souci du beau verbe, appelé de manière ironique par ses principaux détracteurs (les critiques futurs animateurs de la Nouvelle Vague) le cinéma de la « qualité française "; enfin un groupe plus indéterminé, composé de réalisateurs aguerris, non syndiqués pour la plupart, et proposant un cinéma "d'auteur», plus personnel, groupe que nous avons usuellement baptisé "les valeurs sûres du cinéma français ». Quoique volontairement schématique, cette manière de procéder possède d'incontestables avantages puisqu'elle permet de déterminer les grandes tendances privilégiées par l'Etat, en partant de critères de reconnaissance esthétique usuels et acceptés dans le milieu de la critique, de l'enseignement du cinéma et de la tradition cinéphile.

Enfin, nous avons complété cette analyse par l'étude des arrêtés de nomination des membres de la Commission de l'avance sur recettes publiés au Journal officiel et celle des archives de la sous-commission d'agrément conservées à la Bibliothèque du film. Ces dépouillements nous ont permis de mettre à jour l'existence de nombreuses passerelles entre les trois souscommissions chargées de l'agrément, de l'avance sur recettes et de la propagande du film français en France, ainsi que les deux commissions "satellites» (censure et festivals). Ainsi, entre 1960 et 1965, quelques membres de la commission de censure siègent simultanément à la sous- 
commission de l'avance sur recettes : c'est notamment le cas du producteur de la fameuse série des Angélique, Francis Cosne (en 1961) et de Roger Ribadeau-Dumas. Cet ancien producteur-distributeur de films et ancien codirecteur du COIC sous Vichy, élu député de la première circonscription de la Drôme le 6 décembre 1962, rejoint aussitôt la commission consultative du cinéma par arrêté du 15 février 1963. Il sera dès lors le seul à cumuler (au titre de professionnel du cinéma et/ou de représentant du Parlement) les quatre commissions de décision (avance, censure, propagande et sélection pour les festivals). Autre exemple : entre la sous-commission d'agrément (qui intervient au début du processus de création du film) et la souscommission de sélection des films français pour les festivals internationaux (à l'issue de ce processus), on retrouve les mêmes membres: Louis-Emile Galey, Jean Dutourd, Jean Rebotier, Georges Loureau et Roger RibadeauDumas.

Un détail n'échappe pas à la vigilance du chercheur : la plupart de ces membres sont $d$ 'anciens protagonistes du cinéma français des vingt années précédentes, comme Louis-Emile Galey (représentant du cinéma à Vichy, puis directeur du COIC), Maurice Clavel, Francis Cosne (cité plus haut), Jacques Dacquemine, Maurice Bessy, Henri Jeanson et deux anciens codirecteurs du COIC, Roger Richebé et Roger Ribadeau-Dumas ${ }^{28}$. C'est l'organisation même des commissions du cinéma au sein du CNC qui permettait ces jeux de pouvoir. Lorsque Malraux met en place les premiers services du ministère des Affaires culturelles, en 1959, il modifie peu l'organisation d'un CNC héritier d'un mode de fonctionnement où syndicats du cinéma et travailleurs du film jouent depuis la Libération un rôle décisif sur la vie de la corporation.

Découlant de ce phénomène de génération, nombreux sont les cinéastes ayant jadis bénéficié du système de soutien des années 1940 et 1950 qui, en 1960, reprennent le chemin des studios grâce à l'avance sur recettes. Mais l'inverse existe aussi. Nous avons trouvé trace de pressions exercées sur certains cinéastes n'appartenant pas à la corporation ou ne partageant pas ses valeurs. Jean-Luc Godard, dont le film Les carabiniers avait été censuré en 1961, est ainsi prié de solliciter un avis de précensure pour son projet de film Le mépris ainsi que le montre l'arrêté de la commission d'agrément réunie le 21 novembre 196229. Par ailleurs, il n'est pas improbable que les critères économiques d'octroi masquent dans certains cas les a priori politiques des membres de la commission. On peut se faire une idée de ce genre de pratiques à partir de la lecture des comptes rendus de délibérations. Ainsi une société désirant produire en 1962 un film intitulé La guerre d'Algérie se voit contrainte de résoudre avant le premier tour de manivelle d'innombrables tracasseries

28. Le troisième codirecteur du COIC, Robert Buron, étant à la même époque ministre.

29. Procès verbal $n^{\circ} 69$, séance du 21 novembre 1962 (Archives de la sous-commission d'agrément, Bibliothèque du film, Paris). 
administratives (avis de précensure, remboursement de dettes, justification d'apports) qui justifient à elles seules le refus de la commission de lui octroyer un visa. Précisons que ce projet de film, qui aurait été réalisé par un cinéaste sous le pseudonyme de James Blue, ne verra jamais le jour.

\section{0-1965 : une politique du cinéma}

\section{Cinéma et imagerie gaulliste}

L'image de la guerre, et notamment de la seconde guerre mondiale, est encore à l'origine de certains refus ou obstacles à la réalisation de films ou de projets de films dont on peut penser que, dans le contexte du conflit francoalgérien, l'évaluation politique l'emporte sur l'évaluation esthétique. Ainsi Jean Devewer, pacifiste engagé, reçoit en 1961 une enveloppe de 200000 francs (sur scénario) pour son projet de film Les honneurs de la guerre. Mais il ne « joue pas le jeu». Durant le tournage, il modifie son scénario pour en faire un film plus personnel débarrassé de tout esprit de gloriole militaire ou $d$ 'héroïsme national. Le résultat final tranche avec les stéréotypes du film de guerre en vigueur à l'époque et notamment la célébration du peuple français face à l'adversité telle que la codifie le gaullisme partisan depuis la prise du pouvoir par son chef en $1958^{30}$. A la sortie du film, Devewer est ignoré par l'ensemble de la profession (le film est très mal distribué) et le cinéaste sera contraint à la reconversion. L'autre exception est celle d'Armand Gatti, qui reçoit 250000 francs pour L'enclos, un film de 1960 qui, pour un premier essai, traite exclusivement de la vie d'un camp de concentration, sujet jusqu'ici évité par les films subventionnés. Le film est un échec et Gatti, refusé dès lors des dizaines de fois par l'avance sur recettes (un record partagé avec le cinéaste communiste Louis Daquin), ne parviendra jamais à percer dans le cinéma, s'expatriera à Berlin, à Cuba, pour finalement revenir au théâtre de ses débuts. Ces deux exemples permettent d'interroger le lien entre la politique d'aide à la qualité cinématographiques et les représentations officielles de la grandeur nationale.

Si les octrois de subvention nous montrent que l'Etat évite jusqu'en 1963 de financer des films traitant de l'Algérie, il subventionne en revanche les films sur la seconde guerre mondiale - $d$ 'autant que ces films rencontrent souvent un grand succès auprès du public, comme le prouvent les plus de huit millions d'entrées réalisées par La vache et le prisonnier en 1959 et par Un taxi pour Tobrouk en 1961, les confortables recettes de Week-end à Zuydcoote en 1964, les dix-sept millions de spectateurs de La grande vadrouille en 1966 ou

30. Sur cette question, cf. Lindeperg (S.), " La résistance rejouée : usages gaullistes du cinéma ", Politix, 24, 1993. 
encore le succès de Paris brûle-t-il ? en 1966. Les chiffres globaux du cinéma français entre 1957 et 1968 sont par ailleurs révélateurs. D'un côté, l'on assiste à une baisse du nombre de films réalisés sur le thème des guerres de décolonisation (Indochine, Corée, Algérie) : trois en 1957, deux en 1958, aucun pour les trois années suivantes (Le petit soldat est interdit en 1961). De l'autre, le film ayant pour cadre la seconde guerre mondiale connaît un âge d'or. Ces films subventionnés ont pour point commun de reproduire la vision gaulliste $d^{\prime}$ unification des «forces vives» de la nation. Sont scrupuleusement gommés à l'écran les aspects polémiques pour privilégier une vision cinématographique (et idéalisée ?) de la Résistance victorieuse et unie. Dans son ouvrage Guerre et cinéma, Joseph Daniel note à ce propos la présence d'un phénomène de commémoration :

« La relation est évidente entre la guerre d'Algérie et le retour du général de Gaulle au pouvoir. Mais le cinéma sépare nettement ces deux phénomènes [...]. On a rarement vu le lien entre le gaullisme triomphant et la floraison nouvelle de films sur la seconde guerre mondiale [...] Par son chef, ses manifestations et ses rappels d'une continuité historique, le gaullisme remet, en quelque sorte, la seconde guerre "à la mode", comme phénomène-anniversaire ${ }^{31}$. "

Toujours est-il que les films sur la seconde guerre mondiale fleurissent au tournant des années 1960 : un seul en 1957, deux en 1958, huit en 1959, six en 1960, huit en 1961 et sept en 1962, sans compter la déferlante des années 1965-1966 accompagnant les vingt ans de la capitulation allemande (Paris brûle-t-il ?, etc.). Le soutien décisif de la Commission de l'avance sur recettes à ce type de production s'inscrit dans la continuité d'une politique d'Etat tout autant que le partage d'un certain nombre de valeurs par les experts qui composent les commissions. Même si elle n'est pas à l'origine de la production d'un film, l'avance sur recettes en favorise cependant la réalisation. En ce sens, il n'est pas faux d'affirmer que, dans les années 1960, les films les mieux dotés par la politique d'aide au cinéma donnent un reflet assez fidèle des orientations de la politique gaulliste.

\section{Une aide qui soutient les réalisateurs aguerris}

Même si le fait est plutôt minoritaire, cette adéquation entre les films les plus prisés financièrement par la commission et la vision gaulliste de l'histoire illustre indirectement la tendance générale de la politique de l'Etat en matière de soutien à la qualité cinématographique nationale. Car, contrairement à ce qu'une vision traditionnelle enseignait jusqu'ici pour le célébrer ou pour le dénoncer (l'idée d'une avance sur recettes soutenant un jeune cinéma inventif, dynamique et un tantinet contestataire), le premier tableau que nous avons établi en regroupant les réalisateurs bénéficiaires des aides fait apparaître une

31. Daniel (J.), Guerre et cinéma, Paris, Armand Colin, 1972, p. 297. 
nette prédisposition de la Commission de l'avance sur recettes pour le cinéma académique de la "qualité française ", ainsi que pour les cinéastes aguerris au métier (le groupe des "valeurs sûres"). L'analyse des thématiques sociales fait apparaître un goût prononcé pour les scénarios mettant en scène le patriotisme, les valeurs morales et religieuses et le concept de nation. Se dessine enfin un certain académisme (montage linéaire, plans et éclairages travaillés, goût du beau verbe, dialogues ciselés, distribution prestigieuse), notamment pour les films de Delannoy, Dreville et Christian-Jaque.

\section{1) Le cinéma de la « qualité française »}

Nom

Titre

Clair

Delannoy

Lamorisse

Cayatte

Verneuil

Dreville

Ciampi

Joffé (A.)

Beker

Carbonneaux

Allegret (Y.)

Giono

Decoin

De la Patellière

Hunebelle

Christian-Jaque

Autant-Lara
La Française et l'amour

Tout l'or du monde

Les quatre vérités

Les fêtes galantes

Princesse de Clèves

Les amitiés particulières

Le majordome

Voyage en ballon

Fifi la plume

Le passage du Rhin

La vie conjugale de F.

$\mathcal{E} M$.

Cent mille dollars

au soleil

Week-end à Zuydcoote

La sentinelle endormie

La nuit des adieux

Liberté 1

Le ciel sur la tête

Fortunat

Pas question le samedi

Le trou

Gamberge

Germinal

Cresus

La Française et l'amour

Un taxi pour Tobrouk

Fantômas

Le gentleman de Cocody

Journal d'une fermme

en blanc
(1)

(2) Année

Montant de l'aide (anciens francs)

$\begin{array}{cccc}X & & 1960 & 150000 \\ X & & 1961 & 400000 \\ & X & 1963 & 50000 \\ X & & 1965 & 650000 \\ X & & 1960 & 750000 \\ & X & 1964 & 300000 \\ & X & 1965 & 200000 \\ X & & 1960 & 750000 \\ X & & 1964 & 500000 \\ & X & 1960 & 300000 \\ & & 1963 & 400000\end{array}$

X 1964

100000

600000

600000

400000

450000

400000

150000

200000

200000

200000

200000

100000

150000

300000

200000

400000

300000 
2) Les « valeurs sûres » du cinéma français

\begin{tabular}{|c|c|c|c|c|c|}
\hline Nom & Titre & (1) & $(2)$ & Année & $\begin{array}{l}\text { Montant de l'aide } \\
\text { (anciens francs) }\end{array}$ \\
\hline \multirow[t]{3}{*}{ Carné } & Terrain Vague & & $x$ & 1961 & 250000 \\
\hline & Du mouron pour les petits oiseaux & & $x$ & 1963 & 100000 \\
\hline & Trois chambres à Manhattan & $x$ & & 1965 & 200000 \\
\hline \multirow[t]{2}{*}{ Bresson } & Le procès de Jeanne d'Arc & $x$ & & 1961 & 550000 \\
\hline & Au hasard Balthazar & $\mathrm{X}$ & & 1965 & 500000 \\
\hline \multirow[t]{2}{*}{ Melville } & Léon Morin, prêtre & $X$ & & 1961 & 400000 \\
\hline & L'aîné des Ferchaux & $X$ & & 1963 & 150000 \\
\hline \multirow[t]{2}{*}{ Gance } & Austerlitz & & $X$ & 1960 & 600000 \\
\hline & Un contre cent & $X$ & & 1962 & 250000 \\
\hline Astruc & La longue marche & $x$ & & 1965 & 300000 \\
\hline Renoir & Le caporal épinglé & & $\mathrm{X}$ & 1962 & 200000 \\
\hline Bunuel & Journal d'une femme de chambre & & $x$ & 1964 & 100000 \\
\hline Tati & Playtime & $x$ & & 1961 & 950000 \\
\hline
\end{tabular}

L'origine socioprofessionnelle des membres de la commission explique en partie l'anatomie de ce cinéma subventionné durant les cinq premières années d'exercice de l'avance sur recettes. Des choix personnels orientent implicitement les votes, tirant les films bénéficiaires vers une politique qui cache mal ses ambitions de renouer avec le "cinéma de la qualité » qui fit la grandeur des festivals des années 1950. Ces années avaient en effet comblé le cinéma français de prix prestigieux : en 1949, Manon de Clouzot est Lion d'or à Venise ; en 1950, Au-delà des grilles de René Clément est Oscar du meilleur film étranger à Hollywood; Justice est faite est Lion d'or en 1952 ; Jeux interdits remporte un Oscar, de même que Le monde du silence en 1956 et Mon oncle de Jacques Tati en 1958. Ce qui permet de préciser que jusqu'à la fin des années 1970, pour évaluer les retombées des aides d'Etat sur le cinéma, le seul critère utilisé par le CNC reste encore celui de la présence des films aidés au sein des palmarès de festivals internationaux.

Les deux tableaux ci-dessus font par ailleurs apparaître des sous-tendances, comme la "radicalisation" des années 1964 et 1965 . Après analyse des arrêtés de nomination, il ressort que ces années marquent le premier exercice de Pierre Moinot à la présidence de la Commission de l'avance sur recettes ${ }^{32}$. Cette présidence est nettement caractérisée par la sympathie pour

32. Fondateur du système de l'avance sur recettes, $P$. Moinot revient au CNC après trois années passées aux côtés de Malraux, années qui se terminèrent par une brouille avec $\mathrm{E}$. Biasini, arrivé au cabinet en 1961, chargé avec succès de l'organisation des Maisons de la culture et finalement 
les projets présentés par des cinéastes aguerris incarnant la qualité esthétique et culturelle du cinéma français. A compter de la nomination de Pierre Moinot, le 2 avril 1964, nous avons établi la sous-liste des cinéastes ayant bénéficié de sommes supérieures à la moyenne d'une dotation (237 000 francs). Entre cette date et le 31 décembre 1965, un total de soixante cinéastes ont été aidés. Or le sous-total des cinéastes ayant bénéficié des sommes les plus importantes fait à nouveau apparaître les noms de cinéastes distingués dans les deux premiers tableaux établis plus haut (le cinéma de la « qualité française » et les "valeurs sûres " du cinéma français).

$\begin{array}{llll}\text { le } 10 / 06 / 64 & \text { Albert Lamorisse } & \text { Fifi la plume } & 500000 \text { francs } \\ \text { le 15/06/64 } & \text { Yves Ciampi } & \text { Le ciel sur la tête } & 400000 \text { francs } \\ \text { le 09/11/64 } & \text { Christian-Jaque } & \text { Gentleman de Cocody } & 400000 \text { francs } \\ \text { le } 10 / 03 / 65 & \text { Jean Dreville } & \text { La nuit des adieux } & 400000 \text { francs } \\ \text { le } 19 / 03 / 65 & \text { Claude Autant-Lara } & \text { Journal d'une femme en blanc } & 300000 \text { francs } \\ \text { le } 09 / 07 / 65 & \text { René Clair } & \text { Les fêtes galantes } & 650000 \text { francs } \\ \text { le 21/07/65 } & \text { Robert Bresson } & \text { Au hasard Balthazar } & 500000 \text { francs } \\ \text { le 23/08/65 } & \text { Jean Dreville } & \text { La sentinelle endormie } & 600000 \text { francs }\end{array}$

Pour mémoire durant cette même période :

$\begin{array}{llll}\text { le 30/04/64 } & \text { Marcel Carné } & \text { Trois chambres à Manhattan } & 200000 \text { francs } \\ \text { le 08/05/64 } & \text { Henri Verneuil } & \text { Cent mille dollars au soleil } & 100000 \text { francs } \\ \text { le } 12 / 01 / 65 & \text { André Hunebelle } & \text { Fantômas } & 200000 \text { francs } \\ \text { le } 18 / 03 / 65 & \text { Alex Joffé } & \text { Pas question le samedi } & 150000 \text { francs } \\ \text { le } 10 / 05 / 65 & \text { Jean Delannoy } & \text { Majordome } & 200000 \text { francs }\end{array}$

Le cas de René Clair est révélateur de cette radicalisation de la commission puisqu'il obtient plus d'un million de francs pour quatre films réalisés en quatre ans. Robert Cravenne (ancien directeur général d'Unifrance) nous révèle dans sa thèse de droit soutenue à Toulouse en 1995 que René Clair était un intime d'André Malraux, qu'il rencontrait fréquemment en compagnie du président d'Unifrance ${ }^{33}$. De même, Roger Richebé, syndicaliste du cinéma,

nommé directeur de la toute nouvelle direction du théâtre, de la musique et de l'action culturelle.

33. Il est d'autre part établi qu'il entra à l'Académie française en 1960 dès lors que Malraux fut nommé aux Affaires culturelles. René Clair doit également son élection aux milieux intellectuels 
nous apprend dans ses souvenirs ${ }^{34}$ que Michel Fourré-Cormeray, alors directeur du CNC, avait entretenu avant sa nomination des rapports financiers très étroits avec un certain nombre de cinéastes aidés plus tard par l'avance sur recettes. Ainsi Michel Fourré-Cormeray avait produit et distribué dans les années précédant sa nomination à la tête du CNC des films comme $L a$ kermesse hérö̈que de Jacques Feyder, Les diaboliques de Clouzot, Fanfan la tulipe de Christian-Jaque, Le petit monde de Don Camillo de Duvivier, mais surtout de nombreux films de René Clair dont Sous les toits de Paris (1930), Le million (1931) et $A$ nous la liberté (1931).

\section{La genèse de la Nouvelle Vague et l'avance sur recettes}

Pour ce qui concerne les cinématographies écartées par le système, et notamment la Nouvelle Vague, il apparaît, d'après les procès-verbaux conservés à la Bibliothèque du film, que jamais la puissante sous-commission des demandes d'agrément de tournage du CNC n'aurait accepté un film tourné avec du personnel non encarté et un montage financier aléatoire, comme nous le prouvent les refus d'agrément et les difficultés faites à plusieurs films de la jeune génération. Ainsi, un projet soumis par Claude Lelouch en 1963 est refusé pour trois motifs concomitants : « financement non conforme ", " défaut de possession de cartes de la part des techniciens " et "devis sous-estimé ». Contrairement à ce que l'on pensait donc, durant les cinq premières années d'exercice de l'avance sur recettes, six films seulement de la Nouvelle Vague sur le total des 192 bénéficiaires ont le privilège de bénéficier de l'aide. Le tableau ci-dessous en détaille les titres.

\section{3) Le cinéma de la Nouvelle Vague}

\begin{tabular}{|c|c|c|c|c|c|}
\hline Nom & Titre & (1) & (2) & Année & $\begin{array}{l}\text { Montant de l'aide } \\
\text { (anciens francs) }\end{array}$ \\
\hline \multirow[t]{2}{*}{ Truffaut } & La peau douce & & $\mathrm{X}$ & 1964 & 100000 \\
\hline & Jules et Jim & $x$ & & 1962 & 200000 \\
\hline Chabrol & Les bonnes femmes & & $x$ & 1960 & 150000 \\
\hline Godard & Pierrot le fou & & $x$ & 1965 & 250000 \\
\hline \multirow{2}{*}{ Doniol-Valcroze } & Le cour battant & $X$ & & 1961 & 250000 \\
\hline & La dénonciation & $x$ & & 1961 & 250000 \\
\hline
\end{tabular}

de l'époque, qui voyaient en lui le représentant d'un cinéma populaire de qualité, capable d'offrir une image du cinéma français exportable à l'étranger.

34. Richebé (R.), Au-delà de l'écran : 70 ans de la vie d'un cinéaste, Monte-Carlo, Pastorelly, 1977. 
Il s'agit il est vrai, pour quatre d'entre eux (Jules et Jim, La peau douce, Les bonnes femmes et Pierrot le fou), de films importants qui ont largement contribué à la consécration publique de la Nouvelle Vague, au-delà des succès de nouveautés, d'audaces, voire de scandales, des premières années. Ces films ont également permis à leurs auteurs (Chabrol, Truffaut, Godard) d'obtenir la reconnaissance définitive de la profession comme de la critique. Si l'avance sur recettes ne participe en rien à l'émergence de la Nouvelle Vague, elle a contribué à la professionnalisation de ses représentants en les obligeant, s'ils souhaitaient trouver leur place, à se soumettre aux critères de travail en vigueur à l'époque (nombre minimum obligatoire de personnel technique, techniciens dûment encartés, budget et financement conformes, présentation devant la commission du scénario définitif). Il est très révélateur de lire en 1962 le point de vue des Cahiers du cinéma sur le sujet, la revue dénonçant une éviction des cinéastes pour cet ensemble de motifs :

"Notons que le principe de l'Avance relève d'un parti pris esthétique qui condamne par essence certaines esthétiques et en glorifie d'autres. Le film de fiction improvisé, typiquement "Nouvelle Vague", le film où tout repose sur la direction d'acteurs ou la délicatesse des travellings, et qui nous ont donné le meilleur du cinéma, sont handicapés, leur qualité éventuelle ne pouvant être assurée ni même prévue sur le papier. L'Avance contraint donc la Nouvelle Vague au double jeu que constitue la recherche d'alibis littéraires ou thématiques 35 . "

Entre 1960 et 1965, deux des anciens animateurs des Cahiers du cinéma (Rivette et Rohmer) ne trouvent aucun financement. Rivette devra attendre 1968 pour obtenir sa première avance ( L'amour fou) et Eric Rohmer, refusé trois fois par la commission (sur scénario et sur film terminé), attendra 1967 pour obtenir une première participation d'Etat dans La collectionneuse. Les sommes octroyées aux cinéastes de la Nouvelle Vague restent par ailleurs relativement modestes comparées à celles allouées aux autres tendances cinématographiques de l'époque. La popularité de Godard ou de Truffaut ne semble pas avoir joué sur ce plan-là. En effet, l'addition des sommes versées à l'ensemble des cinéastes de la Nouvelle Vague ne parvient pas à égaler celles reçues au titre d'un seul cinéaste, également représentatif du renouveau cinématographique de ces années-là (Alain Resnais par exemple), comme nous le montre le tableau ci-dessous :

35. Interview de Luc Moullet dans Les cahiers du cinéma, 138, 1962 (numéro spécial consacré à la Nouvelle Vague). La question des alibis nés de la contrainte de l'avance est par ailleurs très intéressante et peut expliquer en partie la récupération tardive d'un certain nombre de cinéastes dans le giron du circuit commercial 
Cumul des sommes allouées (tous films confondus) entre 1960 et 1965 :

$\begin{array}{llc}\text { Noms } & \begin{array}{c}\text { Cumul des sommes allouées } \\ \text { (millions d'anciens francs) }\end{array} \\ 1 & \text { Resnais } & 1,50 \\ 2 & \text { Delannoy } & 1,35 \\ 3 & \text { Franju, Lamorisse } & 1,25 \\ 4 & \text { Clair } & 1,20 \\ 5 & \text { Bresson, Etaix } & 1,05 \\ 6 & \text { Dreville } & 1,00 \\ 7 & \text { Tati, Ciampi } & 0,95 \\ 8 & \text { Camus } & 0,90 \\ 9 & \text { Gance } & 0,85 \\ 10 & \text { Y. Robert } & 0,75 \\ 11 & \text { Verneuil } & 0,70\end{array}$

Enfin, parmi les films de la Nouvelle Vague qui bénéficient de l'avance sur recettes, nous ne comptons aucune première œuvre. L'aide à la qualité prend en retard le train d'un mouvement dont les principaux tenants ont déjà bâti réputations et filmographies en dehors des salles des commissions. Les films les plus marquants de la Nouvelle Vague sont par ailleurs sortis sur les écrans français avant janvier 1960, date de la mise en place du système de l'avance sur recettes. René Prédal nous rappelle qu'hormis les deux premiers longs métrages de Claude Chabrol, qui bénéficient du système de l'ancienne "prime à la qualité " instauré en 1955, $A$ bout de souffle, Les 400 coups, Paris nous appartient, Le signe du lion, Ascenseur pour l'échafaud, L'eau à la bouche, Moi un noir, Les liaisons dangereuses, Le bel âge ou Les jeux de l'amour sont produits en dehors de toute intervention financière directe de $1^{\prime}$ Etat $^{36}$.

Les principaux films de la Nouvelle Vague sont nés des capitaux privés de leurs auteurs et de leurs producteurs, c'est-à-dire selon un montage des plus classiques, comprenant parfois une avance du distributeur (notamment pour Truffaut et Chabrol). Pour contourner les obstacles économiques, Truffaut se fixe par exemple un ambitieux objectif : faire de sa maison de production, les Films du Carrosse, une " école artistique " à partir de ses propres revenus, objectif qu'il réalisera en partie avant de se recentrer sur son activité de réalisateur. L'autoproduction exclusive n'est adoptée que par un petit nombre de nouveaux cinéastes comme Mocky, Pollet ou Jossua. La baisse des coûts de production est à mettre au crédit des cinéastes et des

36. Prédal (R.), 50 ans de cinéma français, Paris, Nathan 1995. 
producteurs de la Nouvelle Vague, qui y voient le moyen de limiter les risques financiers liés à l'aventure artistique. Truffaut note :

«La profession s'alarmait devant la crise, mais personne n'envisageait la possibilité de diminuer les devis des films. Car personne, dans la corporation, n'avait intérêt à baisser les prix ${ }^{37}$. "

Le prix de revient des premiers longs métrages de la Nouvelle Vague est en effet dérisoire (autour de cinquante millions d'anciens francs). Dès juin 1960, la revue Esprit pose d'ailleurs la question suivante :

"Les devis modestes des débuts de la Nouvelle Vague sont-ils l'indication de changements, où signifient-ils simplement qu'on ne peut confier des centaines de millions de francs à des réalisateurs qui n'ont pas fait leurs preuves ? Il est incontestable que les "jeunes turcs" du cinéma français ont tenté de créer un nouveau style qui a eu une certaine répercussion sur les conditions économiques de production. [...] La Nouvelle Vague a fait preuve que des films d'un certain ton pouvaient être entrepris sans dépenses extravagantes ${ }^{38}$."

Le circuit financier sur la base duquel se développe la Nouvelle Vague constitue dans un premier temps un modèle assez original d'alternative à la subvention publique. Il laisse dans un deuxième temps la place à l'histoire somme toute banale de réalisateurs aguerris aux rouages financiers de la production, sachant solliciter et obtenir le soutien de l'Etat lorsqu'ils en ont besoin.

\section{Le cinéma national entre tradition et innovation}

Les premières années d'exercice de l'avance sur recettes auraient-elles contribué au financement d'un cinéma national conçu comme un spectacle de qualité certes, mais demeurant un divertissement éloigné des réalités de son temps et des missions d'encouragement esthétique prévues par le législateur? Un élément va dans ce sens: les chiffres des remboursements des films bénéficiaires des avances. Il apparaît que, de 1960 à 1988, 1116 films furent aidés. Sur ce nombre, seuls 106 remboursèrent intégralement l'avance, mais ils sont en revanche 22 à la rembourser intégralement durant les cinq premières années d'exercice de la commission, soit un cinquième de la totalité des films remboursés sur les vingt-huit premières années d'exercice. Cette tendance est confirmée par l'analyse thématique des films aidés. En effet, du film de guerre " classique » (Week-end à Zuydcoote, Verneuil, 1964, 600000 francs ; Un taxi pour Tobrouk, de la Patellière, 1961, 300000 francs) à Tintin et les oranges bleues (Vierne, 1962, 200000 francs) et Le gentleman de Cocody (Christian-Jaque, 1965,

37. France-Observateur, 19 octobre 1961.

38. Esprit, 285, 1960, p. 1006. 
400000 francs), en passant par le film policier (Les tontons flingueurs, Lautner, 1963, 200000 francs), le film d'aventures en costume (Cartouche, de Broca, 1962, 350000 francs) et le grand succès de l'époque avec ses 9,8 millions de spectateurs, La guerre des boutons (Yves Robert, 1962, 250000 francs), l'éventail du «cinéma populaire » français à grand spectacle est honorablement représenté parmi les bénéficiaires d'une aide paradoxalement mise en place "pour faire face aux automatismes fondés sur le seul succès commercial ». Pour avoir confirmation de cette tendance, il suffit de faire la liste les bénéficiaires jusqu'en 1968 : il apparaît que les trois cinéastes le plus souvent aidés jusqu'à cette date sont Yves Robert (Alexandre le bienheureux, Les copains, La famille Fenouillard, La guerre des boutons), Robert Enrico (La belle vie, Au cour de la vie, Les grandes gueules, Ho!, Tante Zita) et Claude Lelouch (L'amour avec des si, Une fille et des fusils, Les grands moments, Un homme et une femme, Vivre pour viore), c'est-à-dire les trois réalisateurs qui généreront le plus d'entrées en salles durant la décade suivante ${ }^{39}$.

Comme un condensé symbolique de la politique du cinéma français des années 1960, la trajectoire et l'image publique de ces trois cinéastes illustrent l'investissement républicain de l'Etat culturel. Ouvrier autodidacte devenu comédien, Yves Robert s'impose comme réalisateur de films à partir du succès considérable et inattendu de La guerre des boutons, adaptation du célèbre roman de Louis Pergaud et mise en scène des valeurs laïques via l'éloge de l'école communale et des milieux populaires français dans leurs aspects les plus chaleureux (c'est-à-dire les plus acceptables du point de vue républicain). Robert Enrico est un pur produit de l'IDHEC, l'Institut des hautes études cinématographiques, une initiative privée d'intérêt public, dépendant à la Libération de la direction générale de la cinématographie (avant d'être placé sous la tutelle du ministère de la Culture) et dont la double mission consiste à former des techniciens nouveaux et à promouvoir le septième art ${ }^{40}$. Dans les années 1950, Robert Enrico est un des réalisateurs de courts métrages civils et militaires les plus productifs et les plus primés ${ }^{41}$. Quant à Claude Lelouch, le succès commercial de ses films malgré le rejet de la critique intellectuelle lui confère une image $d^{\prime}$ " auteur populaire », synthèse parfaitement acceptable aux yeux des tenants de l'Etat culturel, entre l'innovation artistique du moment (la Nouvelle Vague), non dénuée des dérives sectaires propres à la nature inspirée, et le souci de l'intérêt général que manifeste la prise en compte du point de vue du public, c'est-àdire celui des citoyens.

39. Cf. Prédal (R.), «L'Etat-mécène et les créateurs », in La production du cinéma, Grenoble, PUG, 1980.

40. Léglise (P.), Histoire de la politique du cinéma français, tome II, Paris, Pierre Lherminier éditeur, 1977, p. 179.

41. Cf. les notices du Dictionnaire du cinéma, Paris, Larousse, 1986, p. 219 et de L'encyclopédie Roger Boussinot du cinéma, Paris, Bordas, 1989, p. 569. 
Ce bilan des premières années de l'avance sur recettes traduit moins une « prime " à l'académisme qu'une politique de compromis culturel entre un cinéma "traditionnel » et l'innovation artistique du moment, la Nouvelle Vague. Il ne représente donc pas pour autant, comme l'affirme Michel Marie, une traduction institutionnelle des thèses de François Truffaut et des jeunes turcs des Cahiers $d u$ cinéma ${ }^{42}$. Si la conjonction de l'émergence de ce mouvement et de l'élaboration progressive, à la tête de l'Etat, d'une promotion volontariste des films de qualité a contribué à la légitimation et à la consécration de la Nouvelle Vague, il faudra attendre le milieu des années 1980 , et notamment la modification des pratiques de consommation du film - sous l'effet conjugué de la démocratisation culturelle et de la vulgarisation de la compétence artistique via les médias et la prolifération des équipements culturels domestiques ${ }^{43}$ - pour voir le " cinéma d'auteur " s'imposer comme une caractéristique générale du film français de qualité.

42. Marie (M.), La Nouvelle Vague..., op. cit., p. 49.

43. Pour le premier aspect de cette modification, et notamment l'émergence d'une "nouvelle vague de spectateurs", cf. Montebello (F.), "Les intellectuels, le peuple et le cinéma ", in Benghozi (P.-J.), Delage (C.), dir., Une histoire économique du cinéma français (1895-1995). Regards croisés franco-américains, Paris, L'Harmattan, 1997 ; pour le second aspect, cf. Leveratto (J.-M.), La mesure de l'art. Sociologie de la qualité artistique, Paris, La Dispute, 2000, p. 10. 